\title{
The Effect of Particle Size on the Biodistribution of Low- modulus Hydrogel PRINT Particles
}

\author{
Timothy J. Merkel ${ }^{1, \neq}$, Kai Chen ${ }^{1, \neq}$, Stephen W. Jones ${ }^{2}$, Ashish A. Pandya ${ }^{1}$, Shaomin \\ Tian $^{1,7}$, Mary E. Napier ${ }^{3}$, William E. Zamboni ${ }^{4,5,6,7}$, and Joseph M. DeSimone ${ }^{1,5,6,7,8,9,10,11,{ }^{*}}$ \\ ${ }^{1}$ Department of Chemistry, University of North Carolina \\ ${ }^{2}$ Department of Cell and Developmental Biology, University of North Carolina \\ ${ }^{3}$ Department of Biochemistry and Biophysics, University of North Carolina \\ ${ }^{4}$ Institute for Pharmacogenomics and Individualized Therapy, University of North Carolina \\ ${ }^{5}$ Carolina Center of Cancer Nanotechnology Excellence, University of North Carolina \\ ${ }^{6}$ Department of Pharmacology, Eshelman School of Pharmacy, University of North Carolina \\ ${ }^{7}$ Lineberger Comprehensive Cancer Center, University of North Carolina \\ ${ }^{8}$ Institute for Nanomedicine, University of North Carolina \\ ${ }^{9}$ Institute for Advanced Materials, University of North Carolina \\ ${ }^{10}$ Department of Chemical and Biomolecular Engineering, North Carolina State University \\ ${ }^{11}$ Sloan-Kettering Institute for Cancer Research, Memorial Sloan-Kettering Cancer Center
}

\section{Abstract}

There is a growing recognition that the deformability of particles used for drug delivery plays a significant role on their biodistribution and circulation profile. Understanding these effects would provide a crucial tool for the rational design of drug delivery systems. While particles resembling red blood cells (RBCs) in size, shape and deformability have extended circulation times and altered biodistribution profiles compared to rigid, but otherwise similar particles, the in vivo behavior of such highly deformable particles of varied size has not been explored. We report the fabrication of a series of discoid, monodisperse, low-modulus hydrogel particles with diameters ranging from 0.8 to $8.9 \mu \mathrm{m}$, spanning sizes smaller than and larger than RBCs. We injected these particles into healthy mice, and tracked their concentration in the blood and their distribution into major organs. These deformable particles all demonstrated some hold up in filtration tissues like the lungs and spleen, followed by release back into the circulation, characterized by decreases in particles in these tissues with concomitant increases in particle concentration in blood. Particles similar to red blood cells in size demonstrated longer circulation times, suggesting that this size and shape of deformable particle is uniquely suited to avoid clearance.

\footnotetext{
(C) 2012 Elsevier B.V. All rights reserved.

*Corresponding author. Direct correspondence to desimone@unc.edu. Telephone 919-962-2166, fax 919-962-2388. FAuthors contributed equally to this work
}

Publisher's Disclaimer: This is a PDF file of an unedited manuscript that has been accepted for publication. As a service to our customers we are providing this early version of the manuscript. The manuscript will undergo copyediting, typesetting, and review of the resulting proof before it is published in its final citable form. Please note that during the production process errors may be discovered which could affect the content, and all legal disclaimers that apply to the journal pertain. 


\section{Keywords}

hydrogel; deformability; biodistribution; long circulating; biomimetic

\section{Introduction}

Crosslinked hydrophilic polymers called hydrogels possess a number of properties which make them an important class of biomaterials for use in biotechnology and medicine. The high water content of hydrogels makes them soft and rubbery; physical characteristics similar to those of soft tissues[1]. Many hydrogels exhibit excellent biocompatibility, likely as a result of their high water content, causing minimal inflammatory responses, thrombosis, and tissue damage [2-5]. Many hydrogel properties are controlled to a large extent by the crosslink density of the gels, including the swelling, cargo release profile and stiffness of the gels[6, 7]. Because of these properties and their facile control, hydrogels of a range of structures are used in medical applications ranging from drug delivery agents [6,7], to tissue engineering [8] and biosensor coatings [9, 10].

Recently, increasing attention has been directed towards extremely deformable hydrogel particles for their ability to avoid biological filtration mechanisms in model systems and in vitro. Low modulus microgel particles were able to traverse pores which were 1/10 their diameters under pressures relevant to renal filtration, suggesting a clearance mechanism for deformable nanoparticles[11]. Microparticles resembling red blood cells were more readily able to traverse microfluidic channels which mimicked the constrictions of capillaries when crosslink density was lowered[12,13]. Our previous work demonstrated that hydrogel particles composed of lightly crosslinked 2-hydroxyethyl acrylate (HEA), which resembled red blood cells in size and shape, had dramatically altered behavior in vivo in a mouse model due to their flexibility. Particles made from this hydrogel material with a Young's modulus of $7.8 \mathrm{kPa}$ were eliminated from the circulation over 30 times slower than particles which were only moderately (approximately $8 \mathrm{x})$ stiffer $(63.9 \mathrm{kPa})$, presumably due to avoidance of filtration in the capillary beds of the lungs, and to some extent, the spleen[13]. The most elastically soft particles demonstrated the ability to deform to bypass physical barriers encountered in the circulation. Because even slightly less elastic particles (modulus of $16.9 \mathrm{kPa}$ ) had greatly reduced circulation times, a modulus-based threshold for $6 \mu \mathrm{m}$ diameter particles to avoid filtration in vivo was inferred[13]. While flexible hydrogel particles have been shown to have advantageous properties for in vivo applications, relatively few studies have focused on the in vivo behavior of particles with a modulus in this biologically relevant range.

The rigidity of hydrogels is typically tuned by adjusting the amount of crosslinker. While this method provides fairly facile control of hydrogel modulus, increased swelling with decreasing crosslink density results in difficulty in maintaining identical particle size and size distribution with changing crosslink density for most particle fabrication methods[1]. However, with the PRINT ${ }^{\circledR}$ (particle replication in non-wetting templates) method[14, 15], the size and/or shape of the elastomeric molds used in particle fabrication determine the size and shape of the particles. In this study, the in vivo behavior of monodisperse populations of extremely soft hydrogel particles was evaluated as particle size was varied in the micron range while composition and modulus were maintained as constants. We fabricated a series of monodisperse, low modulus $(\mathrm{E}=6.5 \mathrm{kPa})$ hydrogel particles with diameters of $0.8,3.8$, 6.4 and $8.9 \mu \mathrm{m}$. The in vivo circulation profiles and biodistributions of these extremely deformable particles were examined in wild type mice, providing insights into the behavior of such low modulus microgels as particle size varies. 


\section{Materials and Methods}

\subsection{Materials}

Acryloyl chloride (97\%), triethylene glycol (99\%), and 2-carboxyethyl acrylate (CEA, 97\%) were purchased from Sigma-Aldrich and used as received. Photoinitiators 2,2diethoxyacetophenone (DEAP), and 1-hydroxyl-cyclohexyl phenyl ketone (HCPK) were purchased from Aldrich and used as received. Poly(ethylene glycol) diacrylate $\left(\mathrm{PEG}_{4000} \mathrm{DA}\right)$ and methacryloxyethyl thiocarbamoyl rhodamine B (PolyFluor 570) were purchased from Polysciences, inc. and used as received. Dylight 680 maleimide was purchased from Thermo Scientific. All other reagents were purchased from Fisher and were used without further purification unless otherwise noted.

\subsection{Synthesis of Hydroxy PEG Acrylate Monomer}

Mixtures of hydroxy PEG acrylate and hydroxy PEG diacrylate were obtained by reaction of triethylene glycol and acryloyl chloride in anhydrous dichloromethane and triethyl amine, as decribed by Guzman, et al. [16] Briefly, addition of acryloyl chloride proceeded over 3 hours at room temperature and stirred overnight. The salts were removed via filtration and washed with deionized water. The aqueous phase was washed with diethylether to extract the monoacrylate. The ether was removed by rotary evaporator, and the product isolated via column chromatography using a mixture of hexane, ethyl acetate and methanol in a 6:3:1 ratio. Free radical inhibitor 4-methoxy phenol (MEHQ) was added to the product fraction at $500 \mathrm{ppm}$ prior to exhaustive reduced-pressure rotary evaporation yielding a clear, colorless to pale yellow liquid. The acrylic monomer is referred to as hydroxyl PEG acrylate (HPA). Characterization is found in the supplemental information.

\subsection{Modulus Testing}

To measure the mechanical properties of crosslinked HPA hydrogels, macroscopic coupons of the prepolymer mixtures were polymerized via UV light in a Teflon mold. Approximately $650 \mu \mathrm{L}$ of freshly prepared prepolymer mixture was pipette into a $2.5 \times 2.5 \mathrm{~cm}$ square mold. The mold was placed in a low-intensity UV oven, and nitrogen purged for 3 minutes prior to curing with UV light. The cross-linked hydrogels were removed from the mold, weighed, and placed in $\mathrm{pH} 7.4$ phosphate buffered saline (PBS) buffer for 24 hours to fully hydrate. The coupons were patted dry with paper towels and weighed again prior to sectioning into approximately $10 \mathrm{~mm}$ wide strips with a razor blade for testing with an Instron 5556 Universal Testing Machine (Instron) with a strain rate of $5 \mathrm{~mm} / \mathrm{min}$. Young's modulus was determined from the initial slope of the resultant stress-strain curves.

\subsection{Particle Fabrication}

Particles were fabricated using the PRINT process, which has been well described elsewhere $[14,15]$. PRINT molds for these studies were obtained from Liquidia technologies (Research Triangle Park, NC) and consisted of a thin layer of a patterned, photocured perfluoropolyether (PFPE) on top of a flexible poly(ethyleneterephthalate) (PET) backing. For these studies, the pre-polymer mixture was spread onto molds that were chilled to $2-5^{\circ} \mathrm{C}$ on a custom built laminator platform. The reduced temperature prevented evaporation of the pre-polymer solution prior to photo-curing. The filled mold was immediately transferred into a chilled $\left(\sim 5^{\circ} \mathrm{C}\right)$, nitrogen purged UV oven and cured with UV light $(\lambda=365 \mathrm{~nm}, 3$ minutes, power $\sim 20 \mathrm{~mW} / \mathrm{cm}^{2}$ ).

Particles were transferred from the mold by laminating the filled, cured molds to a PET sheet which was coated with a thin film of Luvitec (BASF). The particle-filled mold and Luvitec coating were placed in contact and passed through the nip point of a heated laminator $\left(180^{\circ} \mathrm{F}\right)$. After cooling briefly, the mold was peeled away from the Luvitec sheet, 
leaving the particles on the Luvitec film. Harvesting of the particles into solution was accomplished by dissolving the Luvitec layer in water.

The harvesting process leads to well stabilized particles, but leaves an abundance of dissolved Luvitec in solution. The particles were pelleted via centrifugation at $4{ }^{\circ} \mathrm{C}(12,000$ rpm, 5-10 minutes depending on particle size) and the supernatant removed. The pellet was resuspended in Dulbecco's pH 7.4 PBS buffer (Fisher Scientific) and this procedure was repeated 2-3 times to remove excess Luvitec and any soluble fraction of poly(HPA) or unreacted monomers.

\subsection{Particle Characterization}

To determine particle concentration, thermogravimetric analysis (TGA) was used. A sample of the particle solution was centrifuged exhaustively (14,000 RPM/20 minutes) to pellet the particles. The supernatant $(20 \mu \mathrm{L})$ was pipetted to a tared pan and the weight was monitored on a Perkin Elmer Pyris 1 TGA as the sample was heated and dried, providing the weight of salts and dissolved polymer in solution. In parallel, a $20 \mu \mathrm{L}$ sample of the particle solution was analyzed under identical conditions, with the particle weight determined by subtracting the weight of salts and polymer in solution from this final weight.

Particles dimensions were determined by analysis of images from a microscope mounted camera (Zeiss AxioCam MRm) using Zeiss Axiovision LE software and measuring over 50 particles in pH 7.4 PBS buffer, or by dynamic light scattering (DLS) in the case of $0.78 \mu \mathrm{m}$ particles using a nano ZS zetasizer (Malvern Instruments). Zeta potentials for particles were measured on a nano ZS zetasizer (Malvern Instruments) in water.

\subsection{In vivo Plasma and Tissue Pharmacokinetic (PK) Studies}

PK studies of $0.8,3.8,6.4$ and $8.9 \mu \mathrm{m}$ diameter particles were performed in female BALB/c mice (20-22g). Each particle was administered IV $\times 1$ via a tail vein at $20 \mathrm{mg} / \mathrm{kg}$ (particle dose/body weight). Mice ( $\mathrm{n}=4$ per time point) were sacrificed prior to administration, and at $0.2,0.5,1,3,7,24,56$, and 104 hours after administration of each particle. From each mouse, blood was collected $(\sim 1 \mathrm{~mL})$ in a heparinized tube via cardiac puncture. In addition, lungs, liver, kidneys, spleens, heart and a posterior leg (thigh) muscle were obtained after the blood sample was obtained. Blood and tissue samples were analyzed immediately after collection. Tissues were not perfused.

\subsection{Fluorescent Imaging}

The fluorescent signal from the particles in blood and tissues was measured using an IVIS Lumina fluorescent imager (Caliper Life Sciences) with excitation at $675 \mathrm{~nm}$ and emission measured at $720 \mathrm{~nm}$ according to previously described procedures[13]. Blood was pipetted in $100 \mu \mathrm{L}$ aliquots to black 96 well plates for analysis on the IVIS Lumina imager. Tissues were weighed after imaging, and biodistribution was expressed as a function of fluorescent signal per gram of tissue weight.

\subsection{Pharmacokinetics of Particle Clearance from Blood}

For determination of pharmacokinetic parameters including alpha and beta half-lives, we fit the data to a two-compartment model using nonlinear regression analysis (Excel) [13, 17]. The two compartment model assumes that particles are transported between the central and peripheral compartments with first-order kinetics and that particles are eliminated from the central compartment with first-order kinetics. The data was fit to Equation 1, where $\mathrm{Cp}$ is the concentration of the particle in the plasma, A and B are hybrid coeffecients, $t$ is the time component, and a and $\beta$ are rate constants for the distribution and elimination processes, respectively. 


$$
C p=A e^{-\alpha t}+B e^{-\beta t}
$$

Equation 1

Using standard methods and calculations, we estimated various pharmacokinetic parameters, including at ${ }_{1 / 2}$ (half-life of particle distribution), $\beta \mathrm{t}_{1 / 2}$ (half-life of particle elimination), $\mathrm{V}_{\mathrm{C}}$ (volume of the central compartment), $\mathrm{Vd}_{\beta}$ (volume of distribution during the elimination phase), AUC (area under the curve), and $\mathrm{CL}_{\mathrm{T}}$ (total body clearance).

\subsection{Histological Analysis of Tissues}

Lung, liver and spleen tissues were frozen on dry-ice shortly after harvesting and $10 \mu \mathrm{m}$ frozen sections were prepared for staining. Sections were fixed with $2.5 \%$ paraformaldehyde and stained with $4^{\prime}, 6$-diamidino-2-phenylindole. Sections were imaged with a Zeiss 710 confocal microscope using a $63 x$ oil objective.

\section{Results and Discussion}

\subsection{Low Modulus HPA Hydrogels}

For the fabrication of low-modulus hydrogel particles of different sizes, we synthesized an oligo (ethylene glycol) (OEG) functionalized acrylic monomer. The addition of OEG chains to particles is generally considered useful for in vivo applications and is thought to both increase particle stability and decrease recognition by the reticuloendothelial system[18-20]. However, long side chains reduce the flexibility of the main polymer chain due to increased steric bulk, producing hydrogels that are less flexible, with a higher modulus[7]. To balance these effects, we selected a chain length of 3 ethylene glycol units and attached this to an acrylic monomer. For the determination of mechanical properties, we added varied amounts of a $4,000 \mathrm{~g} / \mathrm{mol}$ PEG diacrylate $\left(\mathrm{PEG}_{4000} \mathrm{DA}\right)$ as a crosslinker and polymerized bulk coupons.

Based on previous work with low modulus hydrogels[13], an elastic modulus of $\leq 10 \mathrm{kPa}$ was targeted for HPA based hydrogels. With deformability similar to that of red blood cells $(15-26 \mathrm{kPa})[21,22]$, a modulus in the target range should be sufficient to show a large effect on particle circulation with $6 \mu \mathrm{m}$ diameter particles. An acid containing monomer (2carboxyethyl acrylate, CEA) was included in the hydrogel monomer mixture at $10 \%$ (by weight) to give the particles a negative charge, thereby decreasing non-specific particle uptake[23]. Bulk samples of HPA hydrogels containing 10\% CEA monomer, 1\% 1hydroxycyclohexyl phenyl ketone photoinitiator and crosslinked with $\mathrm{PEG}_{4000} \mathrm{DA}$ were hydrated in $\mathrm{pH}$ 7.4 PBS to simulate physiological conditions and tested with the Instron tensometer (Table 1). Hydrogels with $0.05 \% \mathrm{PEG}_{4000} \mathrm{DA}$ crosslinker had the desired properties, with a Young's modulus of $6.5 \pm 1.3 \mathrm{kPa}$. This formulation was used to fabricate particles for in vivo testing (Table 2).

\subsection{Fabrication and Characterization of HPA Hydrogel PRINT Particles}

Low-modulus $(\mathrm{E}=6.5 \mathrm{kPa}) \mathrm{HPA}$ hydrogels included $1 \%$ Dylight 680 maleimide for in vivo imaging, and $0.1 \%$ methacryloxyethyl thiocarbonyl rhodamine B for ease of particle analysis during the PRINT process. Both dyes were incorporated into the hydrogel matrix via inclusion in the free radical polymerization reaction that formed the particles. Particles fabricated using discoid PRINT molds with feature diameters of $0.2,1,2$ and $3 \mu \mathrm{m}$, resulted in hydrated diameters of $0.78,3.79 \pm 0.17,6.39 \pm 0.56$, and $8.88 \pm 0.47 \mu \mathrm{m}$ in PBS, respectively (Table 3 ).

Fluorescent microscopy was used to measure the diameter of hydrated particles $(n=50)$ for all except those molded at $0.2 \mu \mathrm{m}$. These particles were too small to accurately measure by 
fluorescence microscopy, but were evaluated using dynamic light scattering to determine their hydrodynamic diameter. The zeta potential of the particles, as measured in water, was approximately $-20 \mathrm{mV}$ in all cases. The particles were suspended in $0.1 \mathrm{wt} \% 2,000 \mathrm{~g} / \mathrm{mol}$ poly (vinyl alcohol) in PBS for in vivo applications. Fluorescent images of the particles can be seen in Figure 1.

\subsection{Pharmacokinetic Disposition in Blood}

The concentration versus time profiles of the $0.78,3.8$ and 6.4 and $8.9 \mu \mathrm{m}$ particles in whole blood are presented in Figure 2. Particle concentration in the blood was determined from blood drawn via cardiac puncture at each time-point. Serial dilutions of particles in freshly drawn mouse blood allowed for the generation of standard curves which correlated fluorescent signal in whole blood to particle concentration. The signal from blood harvested from dosed mice fell within the linear region of these plots in most cases, with late timepoints falling below quantification limits (where the plot deviated from linearity due primarily to interference from autofluorescence in blood) for the case of the $8.9 \mu \mathrm{m}$ particles.

To evaluate the kinetics of clearance for the $0.78,3.8$ and $6.4 \mu \mathrm{m}$ diameter particles, we used a two compartment pharmacokinetic model (Table 4), characterized by a biexponential decrease in particle concentration over time [24], with parameters fit by non-

The fits generated using this method are shown, along with the data, in figure 2. This model, which has often been appropriate to describe the behavior of particles in the circulation[13, 25], describes an initial (distribution) phase where the particles distribute from the plasma into various tissues, followed by a late (elimination) phase that describes the ultimate clearance of particles from the plasma. For small molecules and nanoparticles the distribution is attributed to extravasation into tissue; because of their size, the distribution of our hydrogel microparticles may be due to physical entrapment in constricted capillaries (as in the lung) or tissues with low blood flow (such as the spleen and liver) rather than by an extravasation process.

The clearance profiles were somewhat similar for each of the particles examined, with much of the dose distributing to tissues rapidly after injection and slow elimination half-lives. Elimination half-lives $\left(\beta \mathrm{t}_{1 / 2}\right)$ for the smaller particles, 31 and 60 hours for the 0.78 and 3.8 $\mu \mathrm{m}$ particles, respectively, were shorter than for the red blood cell (RBC) sized $6.4 \mu \mathrm{m}$ particles ( $85 \mathrm{~h}$ ), suggesting that this size may crucial to the long circulation times of RBCs. Additionally, the area under the curve (AUC), was more than 3 times greater for the RBC sized particles than the smaller hydrogels ( 8.5 compared to 2.5 and $1.7 \mathrm{mg} * \mathrm{hr} / \mathrm{mL}$, respectively), indicating that a much greater amount of particles stayed in the circulation over time.

The circulation profile of the largest particles examined in this study $(8.8 \mu \mathrm{m})$ could not be described by a pharmacokinetic model because these were rapidly cleared below the limits of quantification, leaving too few data points for such analysis. These particles did not appear as if their clearance profile would be well described by a standard pharmacokinetic model due to the increase in particle concentration in blood at 3 and 7 hours, the explanation of which may require the application of more complicated models[26]. This effect of increases in particle concentration in the blood at later time points, correlated to decreases in particle signal in either the lungs or spleen in all cases, and occurred reliably in multiple studies. This strongly suggests that these deformable particles are physically trapped in these tissues, and are able to dislodge and re-enter the main circulation before other mechanisms result in irreversible clearance. 


\subsection{Pharmacokinetic Disposition in Tissues}

The disposition of particles in various tissues was examined by evaluation of the particle fluorescence in excised tissues. The smallest particles (hydrodynamic diameter $=0.78 \mu \mathrm{m}$ ) distributed primarily into the spleen and liver after injection. Particle accumulation in these tissues increased after injection, peaking 1 hour after dosing at nearly $80 \%$ of the recovered fluorescence (Figure 3). Clearance to the spleen and liver seems to have been the main mechanism of distribution for these particles, as the initial concentration in the blood dropped rapidly over the first hour post-dose. Accumulation in the lungs, heart and kidneys was minor over the course of the study. The particles were highly concentrated in the spleen, though more total particles were sequestered in the liver. This difference can be seen in comparing figure 3 and figure 4 , which present the biodistribution data on a per tissue and per gram of tissue basis, respectively. After 104 hours, the particles were largely cleared from the blood, with only $3.4 \%$ of the dose still circulating.

Particles with a hydrated diameters of $3.8 \mu \mathrm{m}$ distributed primarily into the spleen and lungs immediately after injection. While signal in the lungs decreased after injection, signal in the spleen increased, peaking at 3 hours and staying fairly constant or slightly decreasing over the next 101 hours. These particles accumulated to some extent in the liver over the course of the study, with signal peaking 24 hours after injection and decreasing slightly thereafter. Liver accumulation was minor when viewed on a per gram basis (Figure 4). Accumulation in the kidneys and heart remained low at all times, decreasing over the course of the scan, possibly due to the small amount of blood remaining in these tissues. Particles were cleared to low, but still measurable, levels in the blood by the final time-point examined, with $4.1 \%$ of the injected dose in the blood at 104 hours.

Particles with $6.4 \mu \mathrm{m}$ diameters distributed primarily into the spleen and lungs immediately after injection with a profile similar to what we observed in previous studies with low modulus particles of this size[13]. Signal in the lungs decreased after injection to low levels. Signal in the spleen and liver increased after injection, with signal in the spleen peaking at 3 hours and the signal in the liver peaking later, 7 hours after injection. Signal in the spleen dipped at the 24 hour time-point, correlating to an increase in signal in the blood. This effect may have been due to the release of physically entrapped particles from the spleen back into the circulation. This trend was observed reliably over several experiments and appears to be characteristic of highly deformable particles of this size. A major function of the spleen is to sequester older or damaged red blood cells based on their decreasing deformability, as demonstrated by their ability to navigate narrow fenestrations in this tissue[27]. Older, stiffer cells spend longer residence times in the macrophage-rich red-pulp, from which they are eventually cleared. The tissue distribution data suggest that these particles, which share similar size, shape and deformability with red blood cells, may be cleared in the same fashion. Accumulation in the kidneys, heart, and muscle tissue remained low at all times, decreasing over the course of the scan, possibly due to the small amount of blood remaining in these tissues. At the final time-point examined, 104 hours post-dose, $13.2 \%$ of the injected dose remained in the blood.

The largest particles, with hydrated diameter of $8.8 \mu \mathrm{m}$, were sequestered primarily in the lungs after injection, likely due to their large size compared to the diameter of the capillary beds in this tissue, and only low amounts of particles remained in the circulation. Particles cleared from the lungs over the course of the study, with accumulation in the spleen and liver increasing over time. Liver accumulation was the most significant for these larger particles compared to the other sizes examined, especially when viewed on a per gram basis (Figure 3). As with the $6.4 \mu \mathrm{m}$ diameter particles, a dip in the amount of particles in the spleen and a corresponding increase in the blood occurred. For these larger particles, this effect was more dramatic, and happened earlier in the study ( 7 hours post-dose). These 
particles were largely cleared from the circulation to below quantifiable levels by 24 hours post injection, correlating to less than $3 \%$ of the injected dose.

Histological sections of lung, liver and spleen tissues were taken from mice sacrificed 104 hours after dosing to determine if the particles remained intact in over the course of the study. Intact particles were detected in the liver and the spleen of mice at the end of the study for all particle sizes (Supplementary figure S1), though only the largest particles were detected in the lungs. A more thorough study of the intraorgan distribution of these particles may further enhance our understanding of the clearance mechanisms for these particles, and will be the subject of future studies.

Conventional wisdom regarding the design of micro- and nano- particles would predict the smallest particles in this study to be the longest circulating ones[28], as they should be able to more easily avoid physical filtration. However, we have observed a much shorter elimination half-life for these $780 \mathrm{~nm}$ particles than for $6.4 \mu \mathrm{m}$ particles ( 31 and 85 hours, respectively). A possible explanation for this relatively rapid clearance is the influence of particle size on the rate of cellular uptake, with smaller particles more rapidly internalized $[29,30]$. It is possible that these smaller particles were more rapidly recognized and phagocytosed by macrophages in the liver and spleen, leading to their more rapid clearance from the circulation. The shorter circulation time of the $3.79 \mu \mathrm{m}$ particles also lends support to this argument, as these microparticles would be expected to more readily avoid sequestration in the fenestrations in the spleen that might entrap the $6.4 \mu \mathrm{m}$ particles due to their marginally smaller size, while still large enough to avoid primary clearance in the liver. Further work will be required to fully understand the different mechanisms of clearance that act on these highly deformable particles as particle size varies.

\section{Conclusions}

Particles with extremely high levels of deformability, characterized by a Young's modulus of $6.5 \mathrm{kPa}$, are able to avoid or escape filtration when injected intravascularly in mice. Evidence of avoidance is clear from the long ( $>30$ hours) elimination half-lives of all but the largest of these particles. Escape from clearance tissues is also clearly seen, as these particles tended to accumulate into the spleen over time, reaching a maximum value then decreasing. The decrease in accumulation in the spleen correlated with an increase in the concentration of particles in the blood, presumably due to the release of some of the physically entrapped particles. This was seen most dramatically in the largest of the microgels studied $(8.9 \mu \mathrm{m})$, where the particle concentration decreased for the first hour of the study before increasing at the 3 and 7 hour time points, presumably due to the escape of particles from the lungs and spleen, which resulted in a corresponding decrease in particle accumulation. Finally, higher circulation persistence and slower clearance were seen for deformable particles that resembled red blood cells in size than for both larger and smaller particles, indicating that this combination of size, shape and deformability results in particles that are better adapted to the avoidance of natural clearance mechanisms in the body.

The results presented here demonstrate the importance of size and deformability for determining the biodistribution and circulation persistence of hydrogel particles. This information is expected to have a large impact on the design of hydrogel particles for therapeutic or diagnostic applications and to provide a foundation for the use of such highly deformable particles for drug delivery.

\section{Supplementary Material}

Refer to Web version on PubMed Central for supplementary material. 


\section{Acknowledgments}

We acknowledge the National Institutes of Health (NIH) Director's Pioneer Award, the Center for Cancer Nanotechnology Excellence, Liquidia Technologies, the Chancellor's Eminent Professorship and NIH (1R21HL092814-01 and 1R21HL096011) for support. Joseph DeSimone is a founder, member of the board of directors, and maintains a financial interest in Liquidia Technologies.

\section{References}

1. Mitragotri S, Lahann J. Physical approaches to biomaterial design. Nat Mater. 2009; 8:15-23. [PubMed: 19096389]

2. Graham NB. Hydrogels: their future, Part II. Med Device Technol. 1998; 9:22-25. [PubMed: 10179196]

3. Graham NB. Hydrogels: their future, Part I. Med Device Technol. 1998; 9:18-22. [PubMed: 10176140]

4. Langer R, Peppas NA. Advances in biomaterials, drug delivery, and bionanotechnology. AIChE Journal. 2003; 49:2990-3006.

5. Nguyen KT, West JL. Photopolymerizable hydrogels for tissue engineering applications. Biomaterials. 2002; 23:4307-4314. [PubMed: 12219820]

6. Hamidi M, Azadi A, Rafiei P. Hydrogel nanoparticles in drug delivery. Adv Drug Deliv Rev. 2008; 60:1638-1649. [PubMed: 18840488]

7. Kabanov AV, Vinogradov SV. Nanogels as pharmaceutical carriers: finite networks of infinite capabilities. Angew Chem Int Ed Engl. 2009; 48:5418-5429. [PubMed: 19562807]

8. Leach JB, Schmidt CE. Characterization of protein release from photocrosslinkable hyaluronic acidpolyethylene glycol hydrogel tissue engineering scaffolds. Biomaterials. 2005; 26:125-135. [PubMed: 15207459]

9. Justin G, Finley S, Abdur Rahman A, Guiseppi-Elie A. Biomimetic hydrogels for biosensor implant biocompatibility: electrochemical characterization using micro-disc electrode arrays (MDEAs). Biomedical Microdevices. 2009; 11:103-115. [PubMed: 18679800]

10. Yu B, Wang C, Ju YM, West L, Harmon J, Moussy Y, Moussy F. Use of hydrogel coating to improve the performance of implanted glucose sensors. Biosensors and Bioelectronics. 2008; 23:1278-1284. [PubMed: 18182283]

11. Hendrickson GR, Lyon LA. Microgel Translocation through Pores under Confinement. Angew Chem Int Ed Engl. 2010; 49:2193-2197. [PubMed: 20183836]

12. Haghgooie R, Toner M, Doyle PS. Squishy Non-Spherical Hydrogel Microparticles. Macromol Rapid Commun. 2009; 31:128-134. [PubMed: 21590884]

13. Merkel TJ, Jones SW, Herlihy KP, Kersey FR, Shields AR, Napier M, Luft JC, Wu H, Zamboni WC, Wang AZ, Bear JE, DeSimone JM. Using mechanobiological mimicry of red blood cells to extend circulation times of hydrogel microparticles. Proc Natl Acad Sci U S A. 2011; 108:586591. [PubMed: 21220299]

14. Merkel TJ, Herlihy KP, Nunes J, Orgel RM, Rolland JP, DeSimone JM. Scalable, Shape-Specific, Top-Down Fabrication Methods for the Synthesis of Engineered Colloidal Particles. Langmuir. 2009; 26:13086-13096. [PubMed: 20000620]

15. Rolland JP, Maynor BW, Euliss LE, Exner AE, Denison GM, DeSimone JM. Direct fabrication and harvesting of monodisperse, shape-specific nanobiomaterials. J Am Chem Soc. 2005; 127:10096-10100. [PubMed: 16011375]

16. Guzman J, Iglesias MT, Riande E, Compan V, Andrio A. Synthesis and polymerization of acylic monomers with hydrophilic ling side groups. Oxygen transport through water swollen membranes prepared from these polymers. Polymer. 1997; 38:5227-5232.

17. Brown AM. A step-by-step guide to non-linear regression analysis of experimental data using a Microsoft Excel spreadsheet. Comput Methods Programs Biomed. 2001; 65:191-200. [PubMed: 11339981]

18. Alexis F, Pridgen E, Molnar LK, Farokhzad OC. Factors affecting the clearance and biodistribution of polymeric nanoparticles. Mol Pharm. 2008; 5:505-515. [PubMed: 18672949] 
19. Niidome T, Akiyama Y, Mori T, Katayama Y. The effects of PEG grafting level and injection dose on gold nanorod biodistribution in the tumor-bearing mice. J Controlled Release. 2009; 139:8184.

20. Niidome T, Yamagata M, Okamoto Y, Akiyama Y, Takahashi H, Kawano T, Katayama Y, Niidome Y. PEG-modified gold nanorods with a stealth character for in vivo applications. J Controlled Release. 2006; 114:343-347.

21. Doshi N, Zahr AS, Bhaskar S, Lahann J, Mitragotri S. Red blood cell-mimicking synthetic biomaterial particles. Proc Natl Acad Sci U S A. 2009; 106:21495-21499. [PubMed: 20018694]

22. Dulinska I, Targosz M, Strojny W, Lekka M, Czuba P, Balwierz W, Szymonski M. Stiffness of normal and pathological erythrocytes studied by means of atomic force microscopy. J Biochem Biophys Methods. 2006; 66:1-11. [PubMed: 16443279]

23. Gratton SE, Ropp PA, Pohlhaus PD, Luft JC, Madden VJ, Napier ME, DeSimone JM. The effect of particle design on cellular internalization pathways. Proc Natl Acad Sci U S A. 2008; 105:11613-11618. [PubMed: 18697944]

24. Neubauer AM, Sim H, Winter PM, Caruthers SD, Williams TA, Robertson JD, Sept D, Lanza GM, Wickline SA. Nanoparticle pharmacokinetic profiling in vivo using magnetic resonance imaging. Magn Reson Med. 2008; 60:1353-1361. [PubMed: 19025903]

25. Gratton SEA, PohhauS PD, Lee J, Guo I, Cho MJ, DeSimone JM. Nanofabricated particles for engineered drug therapies: A preliminary Biodistribution study of PRINT (TM) nanoparticles. J Controlled Release. 2007; 121:10-18.

26. Li M, Al-Jamal KT, Kostarelos K, Reineke J. Physiologically Based Pharmacokinetic Modeling of Nanoparticles. ACS Nano. 2010; 4:6303-6317. [PubMed: 20945925]

27. Bowdler, AJ. Structure, Function, and Clinical Disorders. Humana Press Inc; Totowa: 2002. The Complete Spleen; p. 18-20.p. 30-47.p. 140

28. Davis ME, Chen Z, Shin DM. Nanoparticle therapeutics: an emerging treatment modality for cancer. Nature Reviews Drug Discovery. 2008; 7:771-782.

29. Champion JA, Walker A, Mitragotri S. Role of particle size in phagocytosis of polymeric microspheres. Pharm Res. 2008; 25:1815-1821. [PubMed: 18373181]

30. Perry JL, Herlihy KP, Napier ME, Desimone JM. PRINT: A Novel Platform Toward Shape and Size Specific Nanoparticle Theranostics. Accounts of Chemical Research. 2011; 44:990-998. [PubMed: 21809808] 

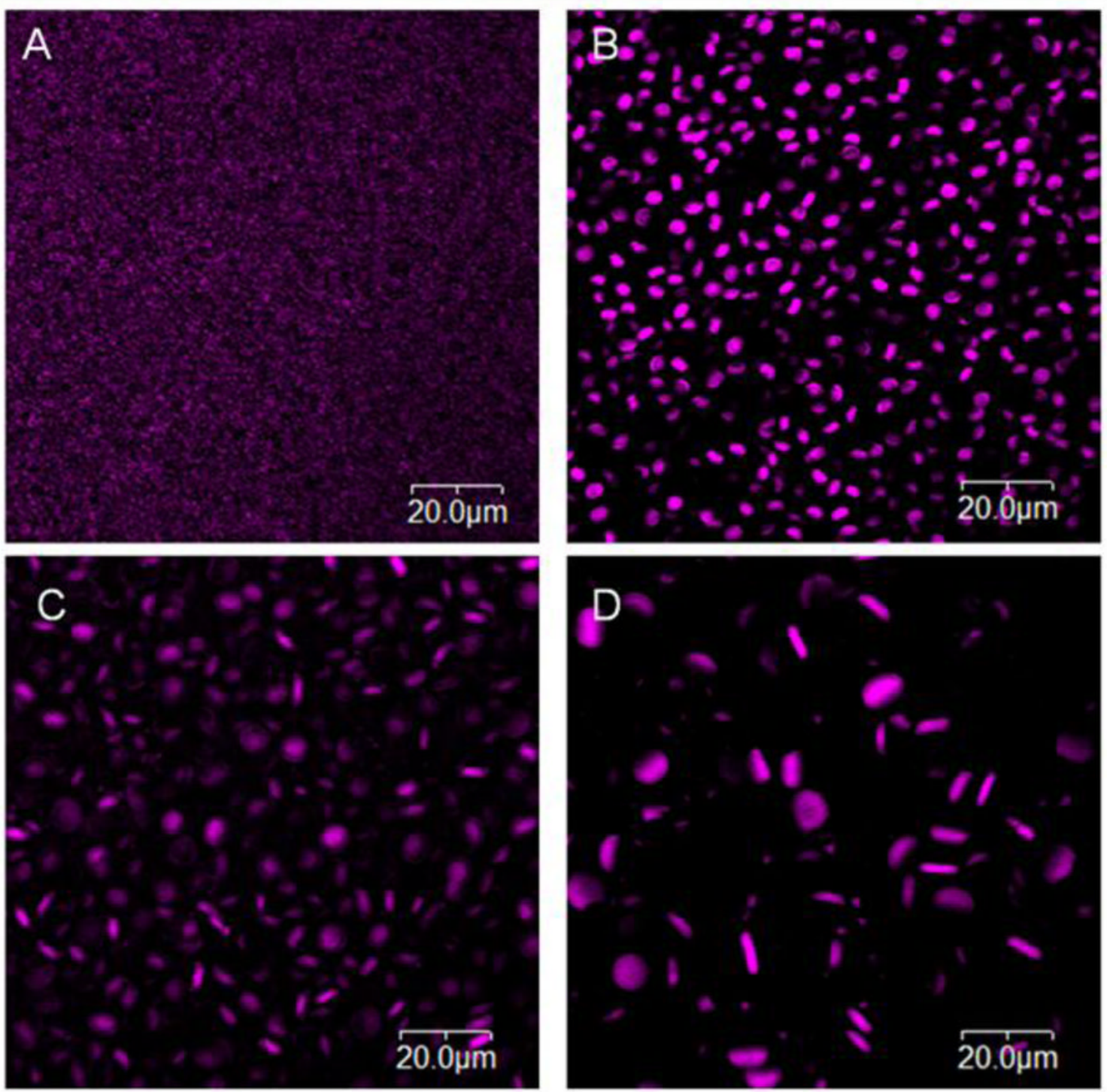

Figure 1.

Fluorescent images of the particles used for the in vivo studies. The particles have hydrated diameters of (A) $0.78 \mu \mathrm{m}$ (B) $3.79 \pm 0.17 \mu \mathrm{m}$, (C) $6.39 \pm 0.56 \mu \mathrm{m}$, and (D) $8.88 \pm 0.47 \mu \mathrm{m}$. Diameters were measured by dynamic light scattering (A) or by analysis of multiple fluorescent images (B-D) using Axiovision LE software. Scale bars are $20 \mu \mathrm{m}$. 


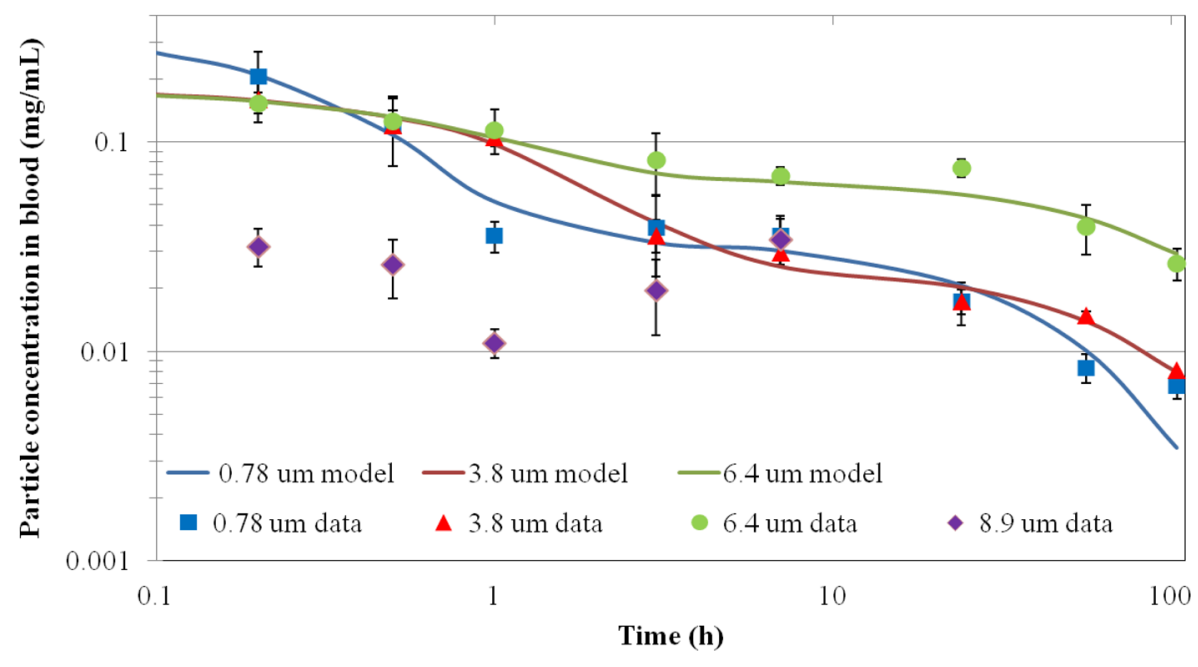

Figure 2.

Change in particle concentration in blood over time for deformable hydrogel particles of different sizes. Curves generated by application of a two-compartment pharmacokinetic model are shown with solid lines. The largest of these particles, with hydrated diameters of $8.9 \mu \mathrm{m}$, were cleared to concentrations below the limits of quantification after 7 hours and were not well-described by the pharmacokinetic model due to increases in concentration before falling under the quantification limit. Each data point represents 4 mice, with error bars representing one standard deviation. linear regression analysis [17]. 


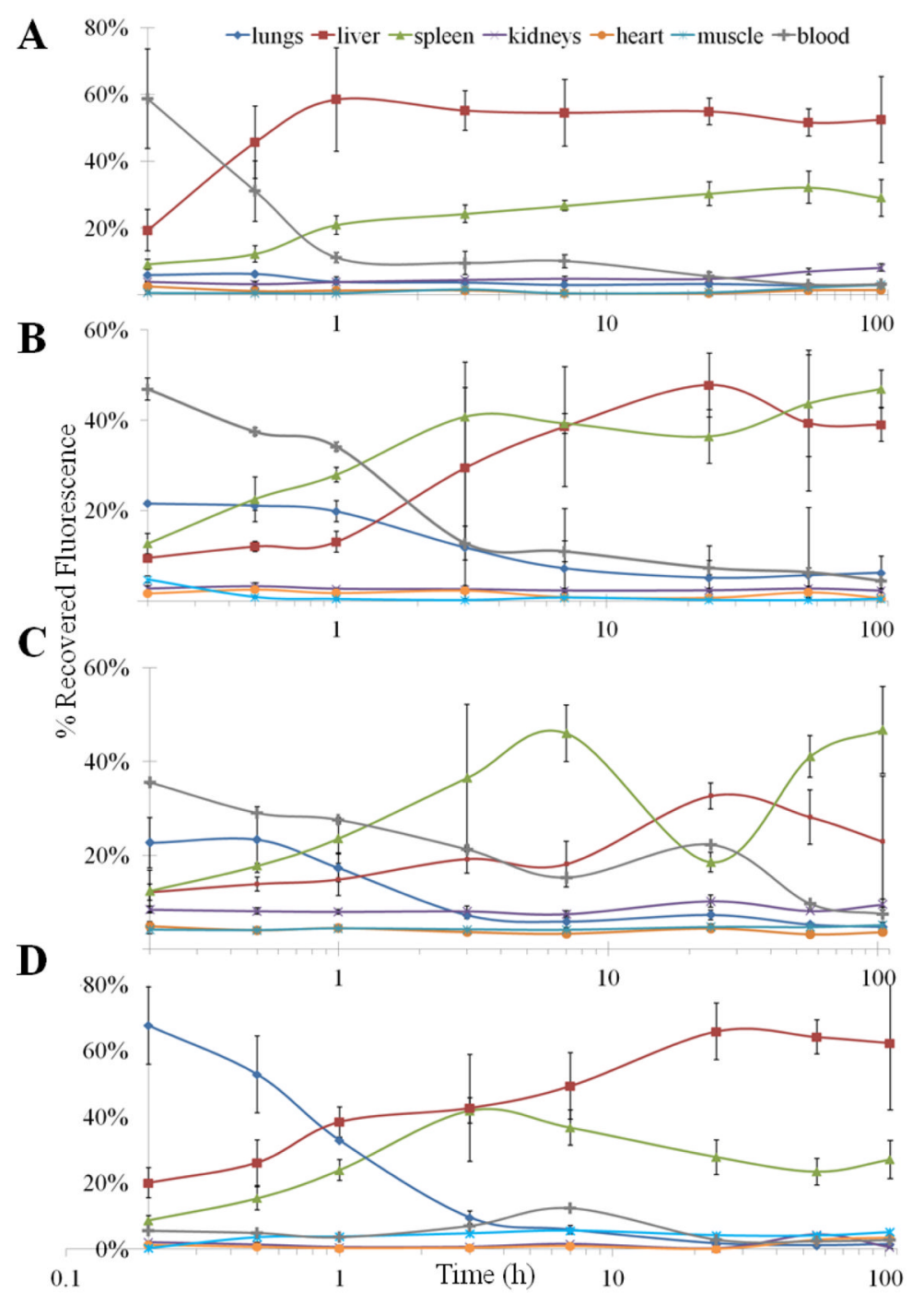

Figure 3.

Semi-log plot showing the biodistribution of highly deformable particles of different sizes in mice over the course of 5 days as measured by the percent of total fluorescence recovered. The fluorescent signal from the particles in each whole tissue examined is shown for particles with diameters of (A) $0.78 \mu \mathrm{m}$, (B) $3.8 \mu \mathrm{m}$, (C) $6.4 \mu \mathrm{m}$ and (D) $8.9 \mu \mathrm{m}$. Lines have been added to connect the data points for clarity only. Four mice were examined per time-point, with error bars representing one standard deviation. 


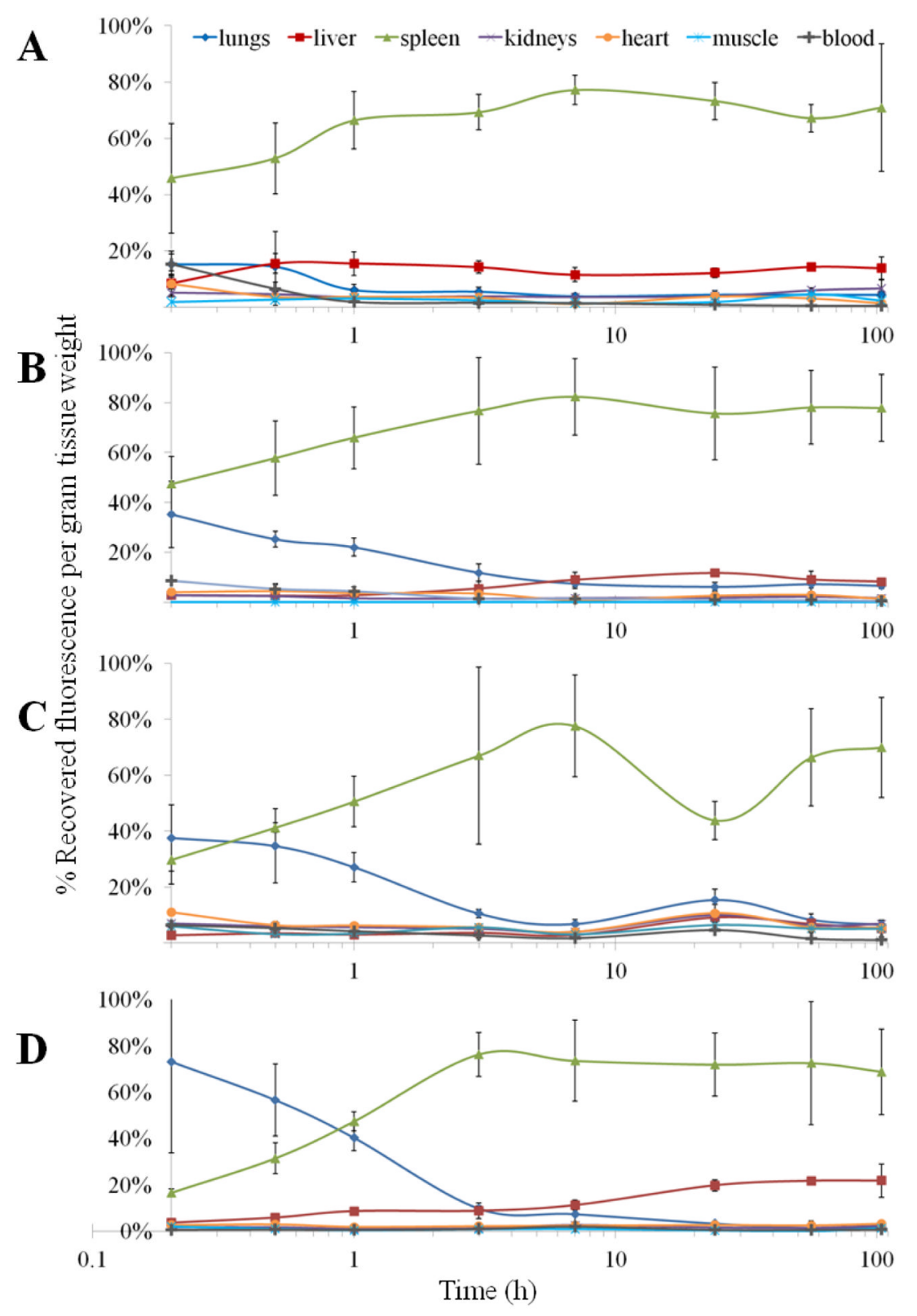

Figure 4.

Semi-log plot showing the biodistribution of highly deformable particles of different sizes in mice over the course of 5 days as measured by the percent of total recovered fluorescent signal from the particles, and adjusted by the weight of each tissue. Data is shown for particles with diameters of (A) $0.78 \mu \mathrm{m}$, (B) $3.8 \mu \mathrm{m}$, (C) $6.4 \mu \mathrm{m}$ and (D) $8.9 \mu \mathrm{m}$. Lines have been added to connect the data points for clarity only. Four mice were examined per time-point, with error bars representing one standard deviation. 


\section{Table 1}

Mechanical properties of hydroxyl PEG Acrylate (HPA) hydrogels with varying amounts of $\mathrm{PEG}_{4000}$ diacrylate crosslinker and $10 \%$ of 2-carboxyethyl acrylate. Swelling ratios were determined from one bulk sample hydrated for 24 hours in PBS. Modulus and strain at break represent an average value determined from three samples cut from the bulk gel, with error representing one standard deviation.

\begin{tabular}{lccc}
\hline \% crosslinker (weight) & $\mathbf{1}$ & $\mathbf{0 . 5}$ & $\mathbf{0 . 0 5}$ \\
\hline E (kPa) & $17 \pm 2.2$ & $13 \pm 1.6$ & $6.5 \pm 1.3$ \\
\% strain at break & $53 \pm 19$ & $46 \pm 7$ & $43 \pm 7.5$ \\
Swelling ratio (wt) & 11.9 & 12.8 & 30.0 \\
Swelling ratio (length) & 2.4 & 2.6 & 3.5 \\
\hline
\end{tabular}


Table 2

The composition of hydrogel particles fabricated for in vivo work.

\begin{tabular}{cc}
\hline Monomer & (\%) \\
\hline HPA & 87.85 \\
2-carboxyethyl acrylate & 10 \\
PEG $_{4000}$ Diacrylate & 0.05 \\
1-hydroxycyclohexyl phenyl ketone & 1 \\
methacryloxyethyl thiocarbonyl rhodamine B & 0.1 \\
Dylight ${ }_{680}$ maleimide & 1 \\
\hline
\end{tabular}




\section{Table 3}

Characterization of particles fabricated for in vivo studies. Particles molded from 1, 2 and $3 \mu \mathrm{m}$ features were measured by microscopy, with error representing one standard deviation from the mean with $n=50$ for particle diameters. Particles molded at $0.2 \mu \mathrm{m}$ were measured by dynamic light scattering to determine their hydrodynamic diameter.

\begin{tabular}{ccc}
\hline Mold diameter & Particle Diameter & Zeta Potential \\
\hline $0.2 \mu \mathrm{m}$ & $0.78 \mu \mathrm{m}(\mathrm{PDI}=0.219)$ & $-17.9 \pm 7.8 \mathrm{mV}$ \\
$1 \mu \mathrm{m}$ & $3.79 \pm 0.17 \mu \mathrm{m}$ & $-22.1 \pm 5.7 \mathrm{mV}$ \\
$2 \mu \mathrm{m}$ & $6.39 \pm 0.56 \mu \mathrm{m}$ & $-17.6 \pm 10.8 \mathrm{mV}$ \\
$3 \mu \mathrm{m}$ & $8.88 \pm 0.47 \mu \mathrm{m}$ & $-22.9 \pm 7.47 \mathrm{mV}$ \\
\hline
\end{tabular}




\section{Table 4}

Pharacokinetic parameters for low modulus hydrogel PRINT particles with increasing diameters. Parameters were determined from particle concentration in whole blood at times $0.2-104$ hours post-dose using a standard two-compartment model.

\begin{tabular}{ccccc}
\hline Particle Diameter $(\boldsymbol{\mu m})$ & & $\mathbf{0 . 7 8}$ & $\mathbf{3 . 7 9}$ & $\mathbf{6 . 3 9}$ \\
\hline $\mathbf{A}$ & $\mathbf{m g} / \mathbf{m L}$ & 0.309 & 0.152 & 0.109 \\
$\mathbf{B}$ & $\mathbf{m g} / \mathbf{m L}$ & 0.0353 & 0.0268 & 0.0682 \\
$\mathbf{a}$ & $\mathbf{h r}^{-\mathbf{1}}$ & 2.88 & 0.76 & 1.08 \\
$\boldsymbol{\beta}$ & $\mathbf{h r}$ & 0.0224 & 0.0116 & 0.00810 \\
$\mathbf{a t}_{\mathbf{1 / 2}}$ & $\mathbf{h r}$ & 0.2 & 0.9 & 0.6 \\
$\mathbf{\beta t}_{\mathbf{1 / 2}}$ & $\mathbf{h r}$ & 31.0 & 59.6 & 85.5 \\
$\mathbf{V}_{\mathbf{C}}$ & $\mathbf{m L} / \mathbf{k g}$ & 1.16 & 2.25 & 2.26 \\
$\mathbf{V d}_{\boldsymbol{\beta}}$ & $\mathbf{m L} / \mathbf{k g}$ & 10.60 & 12.68 & 5.73 \\
$\mathbf{A U C}$ & $\mathbf{m g} \mathbf{h r} / \mathbf{m L}$ & 1.69 & 2.50 & 8.51 \\
$\mathbf{C L}_{\mathbf{T}}$ & $\mathbf{m L} / \mathbf{h r} / \mathbf{k g}$ & 0.24 & 0.16 & 0.047 \\
\hline
\end{tabular}

